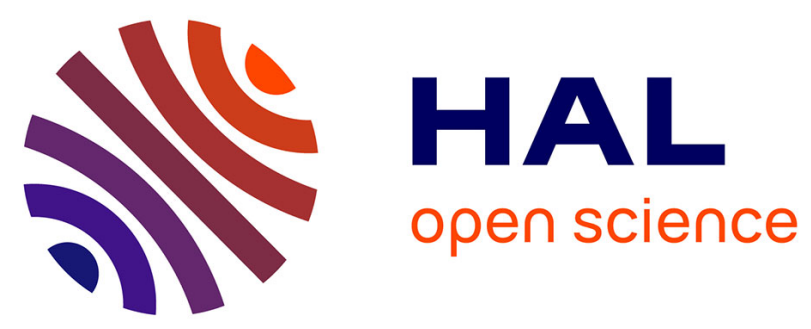

\title{
EXCITATION ACOUSTIQUE IMPULSIONNELLE D'UNE INCLUSION CYLINDRIQUE LIQUIDE
}

\author{
P. Pareige, P. Rembert, J. Izbicki, G. Maze, J. Ripoche
}

\section{To cite this version:}

P. Pareige, P. Rembert, J. Izbicki, G. Maze, J. Ripoche. EXCITATION ACOUSTIQUE IMPULSIONNELLE D'UNE INCLUSION CYLINDRIQUE LIQUIDE. Journal de Physique Colloques, 1990, 51 (C2), pp.C2-391-C2-394. 10.1051/jphyscol:1990292 . jpa-00230714

HAL Id: jpa-00230714 https://hal.science/jpa-00230714

Submitted on 1 Jan 1990

HAL is a multi-disciplinary open access archive for the deposit and dissemination of scientific research documents, whether they are published or not. The documents may come from teaching and research institutions in France or abroad, or from public or private research centers.
L'archive ouverte pluridisciplinaire HAL, est destinée au dépôt et à la diffusion de documents scientifiques de niveau recherche, publiés ou non, émanant des établissements d'enseignement et de recherche français ou étrangers, des laboratoires publics ou privés. 
COLLOQUE DE PHYSIQUE

Colloque C2, supplément au $n^{\circ} 2$, Tome 51 , Février 1990

Ier Congrès Français d'Acoustique 1990

\title{
EXCITATION ACOUSTIQUE IMPULSIONNELLE D'UNE INCLUSION CYLINDRIQUE IIQUIDE
}

\author{
P. PAREIGE, P. REMBERT, J.L. IZBICKI, G. MAZE et J. RIPOCHE
}

Laboratoire d'Electronique et d'Automatique (UItrasons), URA CNRS 1373, Université du Havre, Place Robert Schuman, F-76610 Le Havre. France

\begin{abstract}
Resume - Les experiences présentés consistent en l'examen des fréquences de résonances de la colonne d'eau remplissant une cavite cylindrique situee dans un materiau elastique. Ces resonances sont isalees et identifiees par une nouvelle methode experimentale entierement numerique. Ces résultats sont justifies et comparés a des résultats thériques déja pubifes. Il est montré que ces resonances correspondent a une onde stationnalre dans le diametre de la cavité.

Abstract - The experiments presented consist of the cylindrical cavity set in an elastic matrix. These resonances are isolated and identified by a new and completely numerical method. These results are justified and compared to theoretical results already published. It is shown that these resonances are associated with diametrical stationnary waves.
\end{abstract}

\section{HTRODUCT ION.}

Les auteurs proposent une etude experimentale de la diffusion acoustique d'une cavite circulaire cylindrique situee dans un materiau élastique. La cavite. contient de l'eau et est insonee sous incidence normale par une onde a polarisation longitudinale. La cavite a fond plat de diametre $2 \mathrm{~b}=1 \mathrm{~mm}$ a ete usine au centre d'un bloc d'alfminium cylindrique. Les axes verticaux de la cavite et du bloc sont paralleles. Les transducteurs de contaot utilises sont directionnels, possedent. une entre ces transducteurs et l. alumintum est assure par un gel acoustique. Une nouvelie technique experimentale récemment mise au point au laboratoire est lol utilisee: la M. I.I.R impulsionnelie. C'est une methode d. Isolement et d'Identification des Résonances d'un diffuseur soumis a une excitation Impulsionnelie. Une description detalilee de cette methode est donnee dans la reference [1]. I'Insonation impulsionnelie est effectuee a $1^{\prime a}$ ade d'un transducteur emetteur immobile dont la position sert de reférence angulaire. La reponse est enregistree par l'intermediaire d'un transducteur angulaire. La reponse est enregistree par 1 intermediaire d un transducteur. (Fig.1). Ces contraintes sur les limites du balayage angulaire sont tmposees par la mise en oeuvre du montage [2]. Pour chaque position, les spectres de résonance necessaires a lidentification des résonances de la cavite sont obtenus en traitant par un algorithme, faisant intervenir une Transformee de Fourier Discrete, les signaux diffuses sans leur echo speculaire [3]. Le traitement dé l'ensemble des signaux temporels permet une analyse spectrale des vibrations de la colonne de liquide contenue dans la cavite [4]. Il est ainsi posstble de determiner les frequences de résonance de la colonne de liqulde mais aussi le mode de vibration de ces résonances.

RESULTATS ET DISCUSSIOH.

fréquences r'etude experimentale permet d'isoler 4 résonances dont les sont $3.85,5.34,7.05$ et 8.65 . En comparant les spectres obtenus en retrodiffusion (Fig. ${ }^{34}, \theta=0^{\circ}$; et lorsque les directions des deux retrodiffusion sont perpendiculaires (F1g.2, $\theta=90^{\circ}$ ) on constate que i amplitude de deux raies a fortement decru; ceci laisse supposer pour ces fréquences un mode de vibration impair. Pour ces 4 frequences, les diagrammes angulaires (amplitude du signal diffuse en fonction d'un angle Impulsionnelie (Fig \& 4). Afin de mentrer que l'amplitude des resultats obtenus est sans commune mesure avec le bruit de fond, sur chaque diagramme figure l'évolution du signal diffusé a une frequence non resonnante. Bien que des etudes theoriques deja publiees [5-6] prédisent i existence de resonances de differents modes, les diagrammes angulaires obtenus montrent clairement que seuls certains modes de vibration peuvent etre mesures. Les modes identifies presentent un champ rayonnant exclusivement de type monopolaire au dipolaire. Une verification theorique de ces resultats est donnee par les valeurs de

$J^{\prime} n(k, b)=0$ avec J'm derivee d'ordre $n$ de la fonction de Bessel

En effet cette equation caracterise les frequences propres de la colonne d'eau [5-7]. Les racines de l'equation qui correspondent au probleme sont pour $n=0: 3.83$ et $7.02 ;$ pour $n=1: 5.33$ et $8.54[8]$. 
Pour des raisons deja preclsees, les diagrammes angulaires n'ont pas pu etre releves sur une demi circonference complete. 5 il 1 i y a aucun doute sur l'attribution expertmentale du mode zero a deux des résonances autres. Une verification reste possible en partant du fait etabli que la puissance rayonnee par un dipôle est proportionnelle a la quatrieme puissance rayonne par un dipole est proportionnelle a la quatrieme proportionnelie a la puissance rayonnee est obtenue en calculant liaire sous la courbe de l'amplitude au carré du diagramme angulaire de mode un. Le rapport des valeurs obtenues pour les deux diagrammes est egale à 6.354. Le rapport des frequences a la puissance quatre est $(8.65 / 5.34) 4=6.885$. L'erreur est de $4.3 \%$ et reste trop negligeable pour remettre en cause les hypotheses de depart.

Lors de l'etude de la cavite en retrodiffusion; le signal temporel obtenu en monostatique (un seul transducteur est utilise en émetteur/récepteur> est composé d'un écho spéculaire et d'une suite d'echas rémis d'amplitudes decroissantes. Il a ete etabli un lien entre la suite d'echos et l'allure du spectre de resonances. Fn effet les raies apparaissent sur le spectre separées par un intervalie en frequence qui correspond a 1 inverse du temps necessaire a l'onde longitudinale pour parcourir un aller et retour dans le diametre de la colonne d'eau:

$\Delta \mathbf{A l}=\Delta \mathbf{k}, \mathbf{b} . C_{1} / 2 \mathbf{b} I=C_{1} / 4 \mathbf{b}$ avec $C_{1}:$ celerite de 1 'onde dans $I^{\prime}$ eau.

Cette hypothese est corroboree par le dephasage de II radians qui apparait entre 2 echos successifs de la suite. L'eau etant moins refringente que l'aluminium les rayons refractés generent une caustique a I interieur de la cavite remplie dreau (F1g.5). Des quin rayon touche la caustique sa phase est modifiee de $-\pi / 2$ [9-10]. Ce qui correspond bien a un dephasage de $\pi$ radians pour un aller et retour du rayon refracte dans onde dans le diametre dans la cávité.

La Fig. 6 permet une etude attentive de l'allure du signal temporel enregistre en bistatique pour differentes positions du transaucteur recepteur. A $\theta=0$. le signal diffusée par la cavité présente lialiure recepteurie d un spéculaire et dune suite d echos reems. eses variations den phase des differents echos s'interpretent par le passage par la caustique des rayons refractes. Cette structure du signal tend a disparâtre lorsque $\theta$ approche de la valeur $90^{\circ}$ et à reapparaîte pour des angles de valeur crolssante et superleure a $90^{\circ}$. A $\theta=90^{\circ}$, le signal diffuse par la cavité pleine d'eau apres que celie-ci ait été insonée. ne contient que les 1 informations relatives aux modes 0 . On constate sur la Fintient que les. 1nformations relatives aux modes o. On constate sur la donc ne subit pas 1 'effet de celle-ci.

Il semble qu'11 y ait un lien entre les falts constater sulvants:

Existence des caustiques

Reception d'echos déphases de $\pi$

Existence de mode de vibration $n=0$ et $n=1$

L observation des modes 0 et 1 peut $s^{\prime} i n t e r p r e t e r$ de la façon suivante:

Les sources de vibration existant dans la cavite sont situees sur les frequences de resonance de la cavite, ces sources sont en nombre pair ou impair. A grande distance de la cavité, si le nombre de sources est pair on observe un mode $n=1$; si le nombre de sources est impair, on observe un mode $\mathrm{n}=0$.

\section{CONCLUSION.}

L'ensemble des experiences présentés dans cet article ont permis de determiner. les frequences de resonances de la colonne d'eau remplissant la cavite L'application de la M.I. I. R Impulsionnelie a 1 ietude de la diffusion acoustique par la cavite a permis d'identifler le mode de ces resonances. Les modes ldentifies présentent un champ rayonnant exclusivement de type monopolaire ou dipolaire. Les verifications theoriques corroborent ces resultats. Les varitions de la phase des signaux temporels s'interprete par la creation de caustiques a piinterieur de la cavite. La présence de ces caustiques semble etre a l'origine de i'existence exclusive des deux modes observes.

\section{BIBLIOGRAPHIE.}

[1] Pareige P., Rembert P., Izbicki J.I, Maze G, Ripoche J.,

[2] Pareige P. Rembert P., Maze G., Ripoche J.

Ultrasonics International 89 proceedings, Madrid, a paraitre.

[3] Delestre P. $(1986)$ Ticki J.I., Maze G., Ripoche J.,

[4]. Pareige P., Maze G. Izbicki J.L., Ripoche J.,

1. Acoustique (1988) 1 165-169.

Pao Y.H., Sachse W., J. Acoust. Soc. Am. (1974) 56 1478-1486

[6] Pao Y.H.' Mow C.C.' J. Acoust. Soc. Am. (1976) 59 1046-1056

[7] Maze G., Izbicki J'.L., Ripoche J.

Th congrès françats de mécantque, Bordeaux, actes (1985) 228-229 Kinsler L. E. Frey A.R. Coppens A. B. ' Sanders J.V V.

[9] Griffin J.H, M1klowitz J., Int J Soitds Btruct (1974) 10 1333-1356

[10] KOW.L., $j$. of Applied Mechanics (1970) 37 345-355 


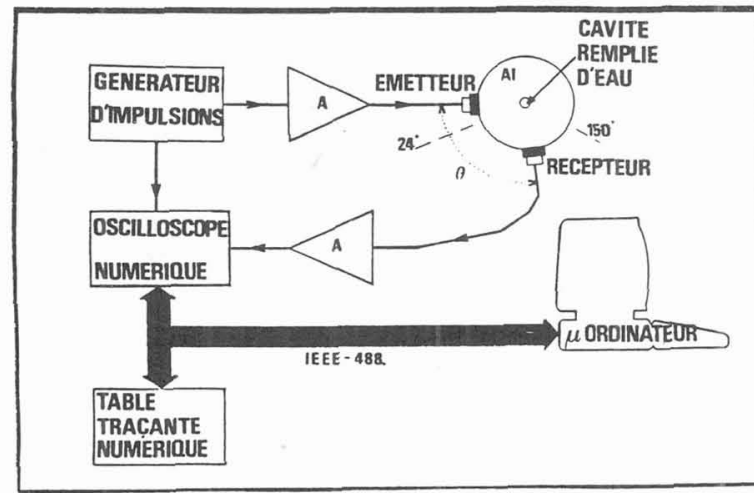

Fig. 1 Dispositif experimental
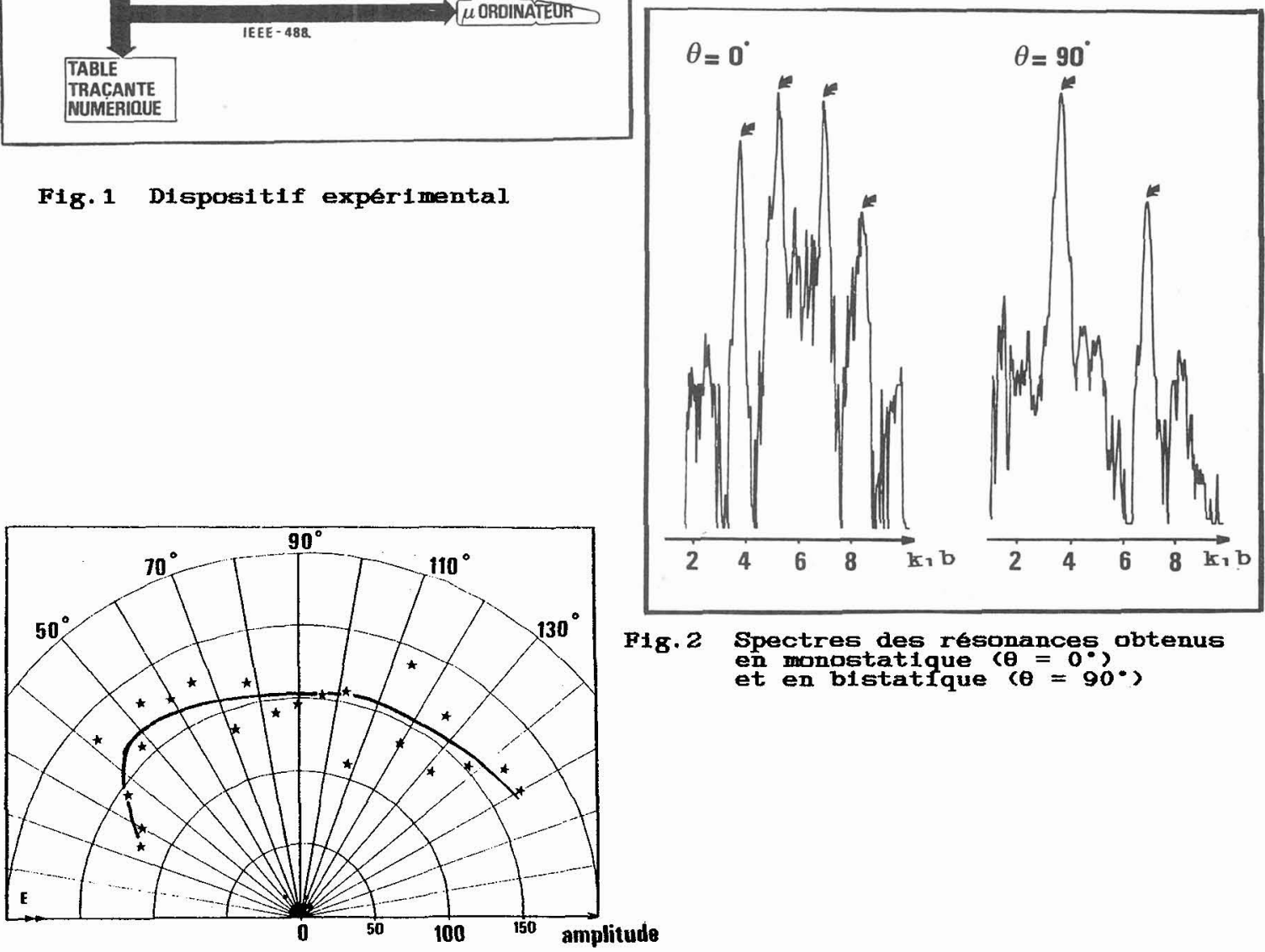

Fig. 2 Spectres des resonances obtenus en mostatique $\left(\theta=0^{\circ}\right\rangle$,
et en bistatique $\left\langle\theta^{\circ}=90^{\circ}\right\rangle$

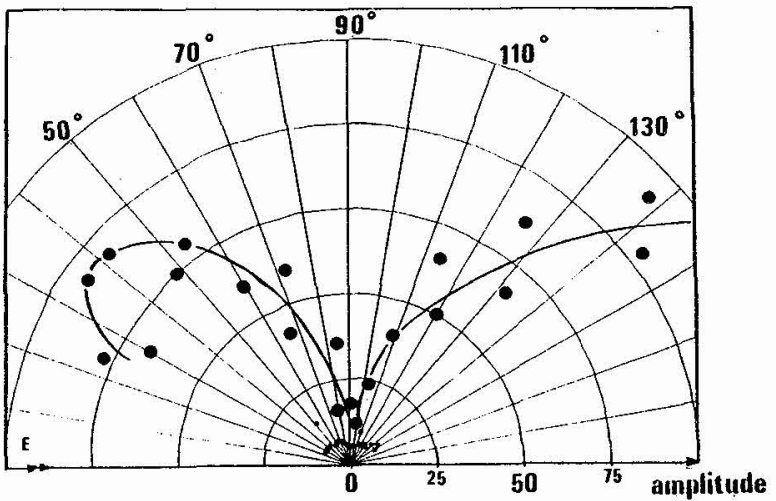

Fig. 3 Identification des résonances aux fréquences réduites $k_{1} b=3.85(\star)$ et $k_{1} b=5.34$ ( $\left.\bullet\right)$ et amplitude hors resonance $(*)$. 

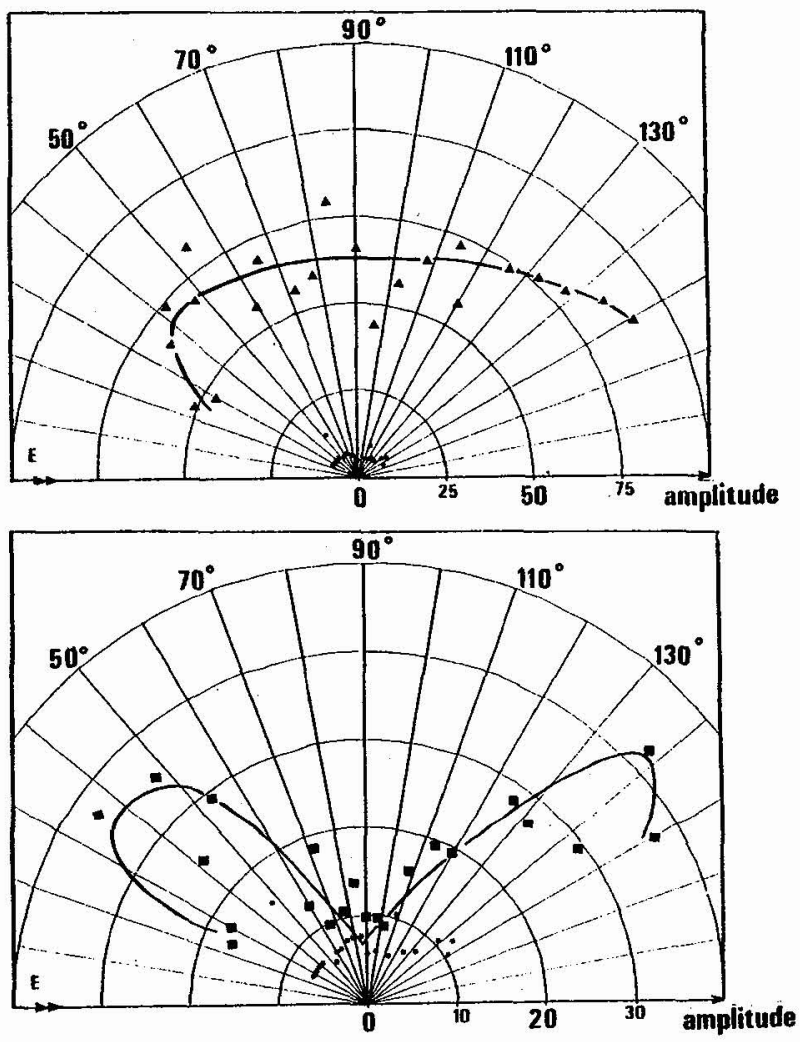

F1g.4 Identification des resonances

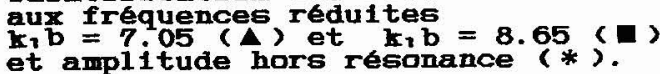
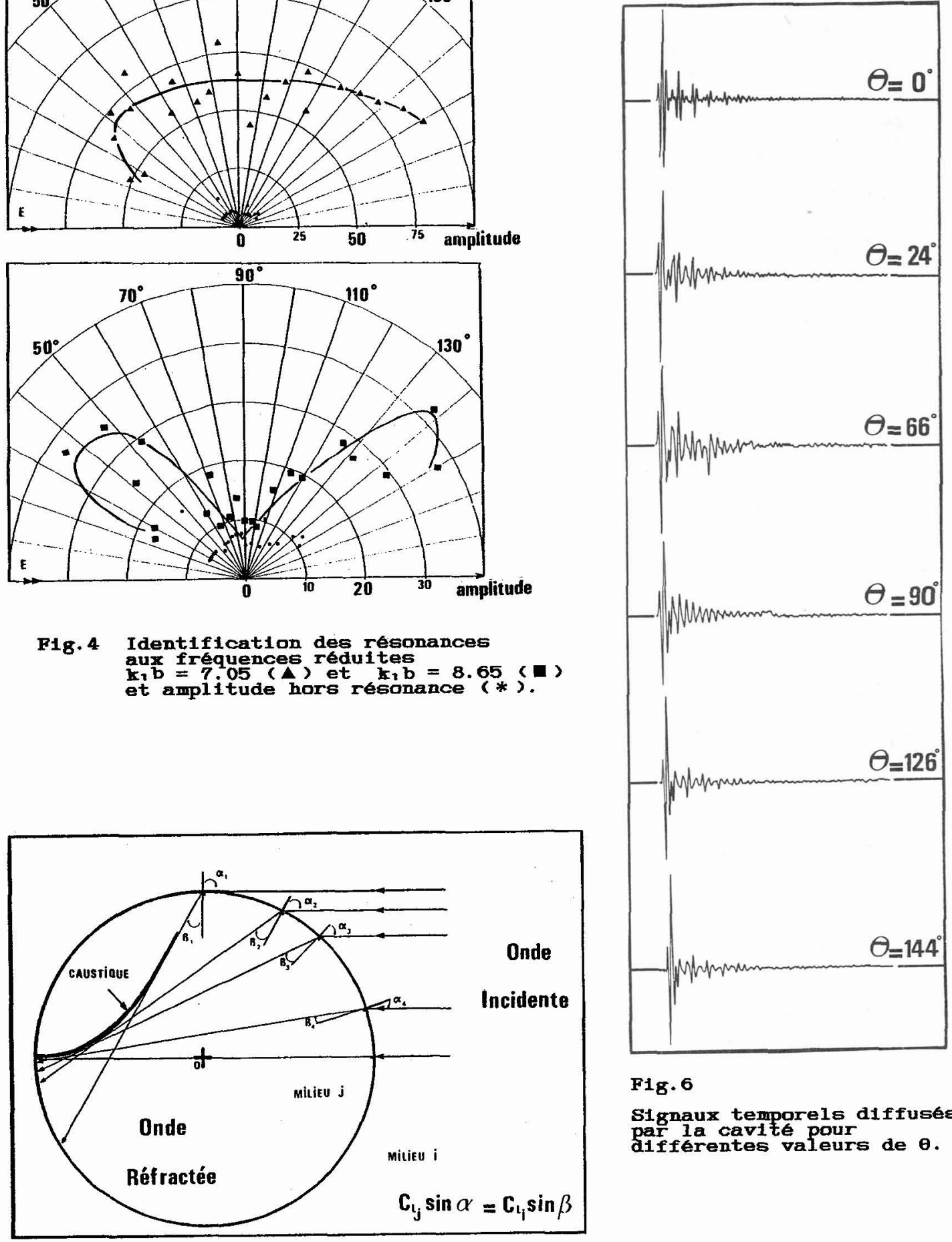

$\mathrm{F1} \cdot 6$

Signaux temporels diffuses par la cavité pour

F1g.5 Tracé des rayons refractes par la cavite et caustique. 